\title{
Final Report On Atomic Database Project
}

\author{
J. Yuan, Z. Gui and G. A. Moses \\ Fusion Technology Institute \\ University of Wisconsin - Madison
}

\section{Introduction}

Atomic physics in hot dense plasmas is essential for understanding the radiative properties of plasmas either produced terrestrially such as in fusion energy research or in space such as the study of the core of the sun. Various kinds of atomic data are needed for spectrum analysis or for radiation hydrodynamics simulations. There are many atomic databases accessible publicly through the web, such as CHIANTI (an atomic database for spectroscopic diagnostics for astrophysical plasmas) from Naval Research Laboratory [1], collaborative development of TOPbase (The Opacity Project for astrophysically abundant elements) [2], NIST atomic spectra database from NIST [3], TOPS Opacities from Los Alamos National Laboratory [4], etc. Most of these databases are specific to astrophysics, which provide energy levels, oscillator strength $f$ and photoionization cross sections for astrophysical elements ( $Z=1-26$ ). There are abundant spectrum data sources for spectral analysis of low $\mathrm{Z}$ elements. For opacities used for radiation transport, TOPS Opacities from LANL is the most valuable source. The database provides mixed opacities from element for $\mathrm{H}(\mathrm{Z}=1)$ to $\mathrm{Zn}(\mathrm{Z}=30)$ The data in TOPS Opacities is calculated by the code LEDCOP.

In the Fusion Technology Institute, we also have developed several different models to calculate atomic data and opacities, such as the detailed term accounting model (DTA) and the unresolved transition array (UTA) model. We use the DTA model for low-Z materials since an enormous number of transitions need to be computed for medium or high-Z materials. For medium and high $\mathrm{Z}$ materials, we use the UTA model which simulates the enormous number of transitions by using a single line profile to represent a collection of transition arrays. These models have been implemented in our computing code JATBASE and RSSUTA. For plasma populations, two models are used in JATBASE, one is the local thermodynamic equilibrium (LTE) model and the second is the non-LTE model. For the LTE model, the calculation is simple since the Boltzmann distribution can be used. As long as we have the energy levels and the ionization energy, we can calculate the plasma population very easily. However, for the non-LTE model, the calculation is very complex since various atomic data are required to build the transition balance matrix. Currently, empirical formulas are used to calculate these data such as electron collision ionization and autoionization. Furnished with these tested atomic data computing codes, we have developed a friendly user interface and a flexible atomic database [5]. 
The UTA model is considered the most practical method for medium and high Z elements since it is very time-consuming and difficult to calculate the enormous number of the transitions. However, the UTA model may overestimate the opacity, therefore, the DTA model is desirable even for medium and high Z elements. With the constant decrease in the cost of the disk storage and increase of CPU speed, it is possible to apply the DTA model to the medium and high Z elements. In this project, we calculate opacities for high Z elements in fully detailed term accounting model for significant populated states. For the various rate coefficients, we calculate the data using the detailed configuration accounting approximation.

In order to handle the large volume of data generated for medium to high- $\mathrm{Z}$ atoms, we use the HDF data format as our database format, which is becoming a standard for storing scientific data.

We have built a sophisticated graphical user interface using Java technology to distinguish our atomic database from other existing databases. Unlike other atomic databases, in which the users can obtain the opacity data in a pair of photon energy and opacity, in our database the user can browser more detailed atomic data information other than the opacity data set by combining our atomic database and Java technology. For example, the user can find out the abundant ion stage and electron configuration state in a certain plasma condition by several clicks on the user interface.

\section{Relativistic atomic model}

The Dirac-Coulomb Hamiltonian for an N-electron atom in the relativistic atomic theory is written as

$$
H=\sum_{i=1}^{N}\left\{c \sum_{i=1}^{3} \alpha_{i} p_{i}+(\beta-1) c^{2}+V(r)\right\}+\sum_{i=1}^{N-1} \sum_{j=i+1}^{N}\left|r_{i}-r_{j}\right|^{-1} .
$$

A configuration state function $|\gamma \mathrm{PJM}\rangle$ of an N-electron system is formed by taking linear combinations of Slater determinants constructed from the oribitals $|\mathrm{n} \kappa \mathrm{m}\rangle$. The multiconfiguration interaction Dirac-Fock method builds the matrix of the Hamiltonian with respect to a basis of configuration state functions. The energy structure is the result of the eigenvalue problem of the Hamiltonian matrix,

$$
\left(H-E_{\Gamma} I\right) c_{\Gamma}=0 \text {, }
$$

where the eigenvalues are the atomic energy levels and the eigenvectors are the mixing coefficients for the configuration states.

For medium or high Z elements, the $d$ and $f$ orbitals are partially filled. This may generate hundreds of relativistic state configurations for one electron configuration under the JJ coupling scheme. The photon transition from atomic state function $\Gamma_{\mathrm{i}}$ to atomic state function $\Gamma_{\mathrm{j}}$ abides by the electric and magnetic multi-pole rules, denoting by

$$
f_{i-j}=\frac{\pi c}{(2 L+1) \omega^{2}}\left|\left\langle\Gamma_{i} P_{i} J_{i}\|O\| \Gamma_{j} P_{j} J_{j}\right\rangle\right|^{2} \text {. The number of allowed transitions can be very }
$$

large. The detailed term accounting method calculates these allowed transitions line by line. 
The approximation applied to calculate this large number of transition array is the UTA model, in which a single line profile is used for this unresolved array. In the RSSUTA model, the configuration averaged oscillator strength is calculated by:

$$
\begin{aligned}
& f_{i c c^{\prime}}=q_{\alpha}\left(1-\frac{q_{\beta}}{g_{\beta}}\right) f_{\alpha \beta}, \\
& f_{\alpha \beta}=\frac{2 m}{\hbar \omega g_{\alpha}} \frac{1}{2 k+1}|<\alpha\|T\| \beta>|^{2}
\end{aligned}
$$

A UTA width is also calculated for the full width at half maximum (FWHM). Figure 1 gives an example of transition lines calculated by the DTA method and the UTA method for transition $3 d^{8} 4 p-3 d^{8} 4 s$ for $\operatorname{Kr}(Z=36)$ [6].

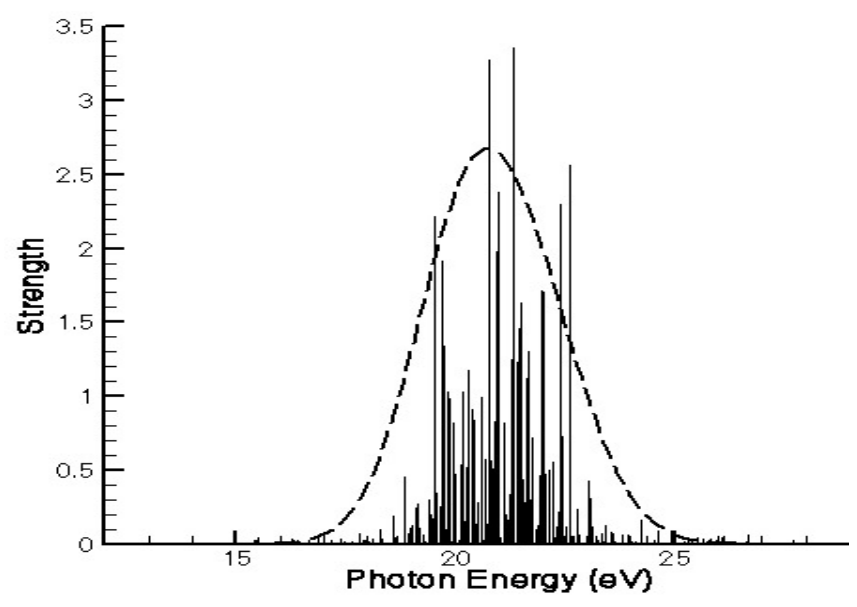

Figure 1. Example of results calculated by the DTA method and the UTA method

We can see from the figure that the UTA method gives a good approximation to the lineby-line DTA calculations. However, it may overestimate the opacity calculation as we can see the area under the UTA profile is much larger than the area covered by the detailed lines. We propose to calculate opacities using the DTA model even for high Z elements.

\section{Outcome of research}

The atomic data involves various kinds of atomic processes. There are a dozen computing tasks in the code. We have completed all tasks proposed in the proposal, including building the electron configuration database; building the configuration state function database for DTA calculations; calculation of bound orbital waves; calculations of free orbital waves; one-electron radial integrals; two-electron radial integrals; configuration-interaction relativistic detailed energy level calculations; photon transitions for the DTA model; photon ionizations; electron collision excitations; electron collision ionizations and autoionizations. All output data are stored in the HDF data format, which can be visualized by the graphical user interface. 
The calculation of the photo-ionization is done under the single-shell approximation and the self-consistent central field, and the calculation of the electron collisions use the factorization method, which is also used in the HULLAC code [7]. For simplicity, the average-configuration approximation is used to calculate the direct and exchange matrix elements.

The cross section for collision excitation in the one-electron approximation is written as

$$
Q\left(n_{a} l_{a} j_{a}-n_{a}^{\prime} l_{a}^{\prime} j_{a}^{\prime}\right)=\frac{8 \pi a_{0}^{2}}{\left(2 j_{a}+1\right) k^{2}} \sum_{k} \sum_{l, l^{\prime}} \sum_{j, j^{\prime}} P^{\lambda}\left(n_{a} l_{a} j_{a}-n_{a}^{\prime} l_{a}^{\prime} j_{a}^{\prime}\right)
$$

where $P^{\lambda}\left(n_{a} l_{a} j_{a}-n_{a}^{\prime} l_{a}^{\prime} j_{a}^{\prime}\right)$ is the summation of the direct matrix element $D^{\lambda}\left(n_{a} l_{a} j_{a} \varepsilon j-n_{a}^{\prime} l_{a}^{\prime} j_{a}^{\prime} \varepsilon^{\prime} l^{\prime} j^{\prime}\right)$ and the exchange element $E^{\lambda}\left(n_{a} l_{a} j_{a} \varepsilon l j-n_{a}^{\prime} l_{a}^{\prime} j_{a}^{\prime} \varepsilon^{\prime} l^{\prime} j^{\prime}\right)$,

$$
\begin{aligned}
& P^{\lambda}\left(n_{a} l_{a} j_{a}-n_{a}^{\prime} l_{a}^{\prime} j_{a}^{\prime}\right)=(2 \lambda+1)^{-1} D^{\lambda}\left(n_{a} l_{a} j_{a} \varepsilon l j-n_{a}^{\prime} l_{a}^{\prime} j_{a}^{\prime} \varepsilon^{\prime} l^{\prime} j^{\prime}\right)\left\langle j_{a}\left\|C^{\lambda}\right\| j_{a}^{\prime}\right\rangle\left\langle j\left\|C^{\lambda}\right\| j^{\prime}\right\rangle \\
& \quad+\sum_{t}(-1)^{\lambda+t}(2 \lambda+1)^{1 / 2}\left\{\begin{array}{rrr}
j_{a} & j^{\prime} & t \\
j & j_{a}^{\prime} & \lambda
\end{array}\right\} E^{\lambda}\left(n_{a} l_{a} j_{a} \varepsilon l j-n_{a}^{\prime} l_{a}^{\prime} j_{a}^{\prime} \varepsilon^{\prime} l^{\prime} j^{\prime}\right)\left\langle j_{a}\left\|C^{\lambda}\right\| j^{\prime}\right\rangle\left\langle j\left\|C^{\lambda}\right\| j_{a}^{\prime}\right\rangle
\end{aligned}
$$

The collision cross sections under the average-configuration approximation are then calculated by taking into account of the electron orbital occupation.

A graphical user interface (see a screenshot in Figure 2) has been developed for atomic presentation. The user interface can plot the transition lines for a certain photon energy range, search the electron configurations generating the user specified transition lines, and calculate opacities. This greatly benefits the Fusion Technology Institute research programs and atomic data user community.

\section{Reference:}

1) CHIANTI: An Atomic Database for Spectroscopic Diagnostics of Astrophysical Plasmas, web site: http://wwwsolar.nrl.navy.mil/chianti.html

2) TOPbase: The Opacity Project at CDS, website: http://vizier.u-strasbg.fr/OP.html

3) Atomic Spectroscopy Data, web site: http://physics.nist.gov/PhysRefData/contentsatomic.html

4) TOPS Opacities, web site: http://www.t4.lanl.gov/cgi-bin/opacity/tops.pl

5) J. Yuan, D. A. Haynes, R. R. Peterson and G. A. Moses, "Flexible Database-driven Opacity and Spectrum Calculations", JQSRT, 81 (2003) 513-520

6) J. Yuan, "High performance computation and database for radiative properties with an interface for ICF applications", PhD Thesis, December 2001.

7) A. Bar-Shalom, M. Klapisch and J. Oreg, Phys. Rev. A, 38 (1988) 1773. 
Figure 2. A Screenshot of YAC Graphical User Interface

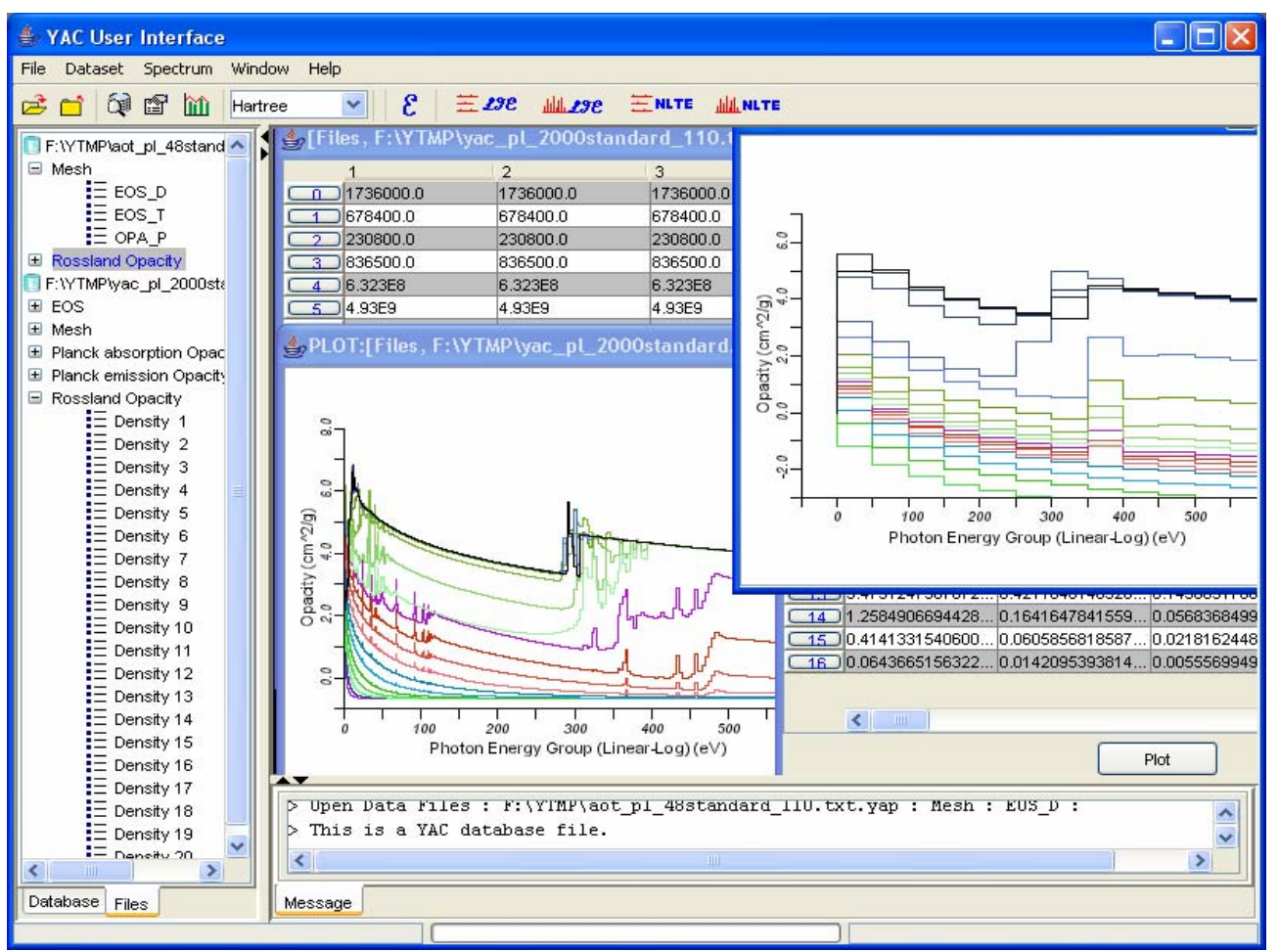

